\title{
An Advanced Embedded SRAM Cell with Expanded Read/Write Stability and Leakage Reduction
}

\author{
Yeonbae Chung*
}

\begin{abstract}
Data stability and leakage power dissipation have become a critical issue in scaled SRAM design. In this paper, an advanced $8 \mathrm{~T}$ SRAM cell improving the read and write stability of data storage elements as well as reducing the leakage current in the idle mode is presented. During the read operation, the bit-cell keeps the noise-vulnerable data 'low' node voltage close to the ground level, and thus producing near-ideal voltage transfer characteristics essential for robust read functionality. In the write operation, a negative bias on the cell facilitates to change the contents of the bit. Unlike the conventional $6 \mathrm{~T}$ cell, there is no conflicting read and write requirement on sizing the transistors. In the standby mode, the built-in stacked device in the $8 \mathrm{~T}$ cell reduces the leakage current significantly. The $8 \mathrm{~T}$ SRAM cell implemented in a $130 \mathrm{~nm}$ CMOS technology demonstrates almost $100 \%$ higher read stability while bearing $20 \%$ better write-ability at $1.2 \mathrm{~V}$ typical condition, and a reduction by $45 \%$ in leakage power consumption compared to the standard $6 \mathrm{~T}$ cell. The stability enhancement and leakage power reduction provided with the proposed bit-cell are confirmed under process, voltage and temperature variations.
\end{abstract}

Key words: SRAM, embedded memory, 8T cell, data stability, leakage current

\section{Introduction}

Technology scaling and increasing random variation in MOSFET characteristics reduce the operational margin of SRAM functionality. Most of current SRAM designs employ the 6-transistor (6T) memory bit-cell composed of two cross-coupled inverters with a set of access transistors. In this traditional 6T SRAM cell, the transistor strength ratios must be chosen such that the read static noise margin (SNM) and the write margin (WM) are both maintained, which presents conflicting constraints on the cell transistor strengths. This

* School of Electronics Engineering,

Kyungpook National University

* This research was supported by Kyungpook National University Research Fund, 2012. This research was also supported by Basic Science Research Program through the National Research Foundation of Korea (NRF) funded by the Ministry of Education, Science and Technology (2011-0003098). The CAD tool was supported by the IC Design Education Center.

Manuscript received Jul. 17, 2012; revised Sep. 26, 2012; accepted Sep. 26, 2012 delicate balance of transistor strength ratios can be severely impacted by device variation, which dramatically degrades the cell operating margin in scaled technologies. Low supply voltages further exacerbate the problem since the threshold voltage variation consumes a large fraction of these voltage margins.

To overcome this stability issue, many different structures of SRAM bit-cell have been explored. For examples, the $7 \mathrm{~T}$ cell [1] may improve the read stability by cutting off a pull down path during read operation. But it has a limited write capability due to the single-ended write operation. The $8 \mathrm{~T}$ [2-4], 9T [5] or 10T [6, 7] SRAM cells decouple the data storage elements and the data output elements, and hence making the read SNM equal to the hold mode SNM. Write-ability is equal to that of the $6 \mathrm{~T}$ cell. However, they do not have efficient column-interleaving structure in the write operation, which might be critical to cope with multi-bit errors. They also might suffer an access time degradation due to the single-ended read-bitline 
structure $[2-4,6,7]$. A data-aware SRAM cell [8], which circumvents the column-interleaving issue in the write operation, consumes a substantial dynamic power because all the non-selected wordlines in the memory array should be toggled during each read and write access. Another 8T cell [9] achieves an exceptional improvement in the read stability, but there is a difficulty in the write operation for low supply voltage. The other cell options $[10,11]$ may increase the read stability, but they carry considerable area penalty in the cell size and hence making unacceptable for most applications.

In this work, improvements of the previous 8-transistor (8T) SRAM cell [9] are addressed. The proposed cell not only enhances the read stability, but also increases the write-ability of the cell inverter pair under voltage variations. It utilizes a differential swing in the read and write path. A column by column negative bias scheme leads to improve the write-ability. There is no column-interleaving issue in the write operation. Furthermore, the bit-cell achieves a significant reduction of the standby current due to the stacked leakage path. All the results in this paper were obtained in a $130 \mathrm{~nm}$ generic CMOS technology. Typical threshold voltages of NMOS and PMOS are $0.34 \mathrm{~V}$ and $-0.34 \mathrm{~V}$, respectively. The nominal supply voltage for this process is $1.2 \mathrm{~V}$.

\section{Proposed SRAM Bit-Cell}

Fig. 1(a) shows a standard 6T SRAM cell, where MN1 and MN2 are the drive, MP1 and MP2 are the load, and MN3 and MN4 are the access transistors, respectively. The fundamental stability issue of this $6 \mathrm{~T}$ cell occurs during the read access. For the read operation shown in Fig. 1(b), the wordline (WL) makes a transition from low to high while the bitline pairs (BL, /BL) are maintained high. The internal node of the cell that represents a zero gets upward through the access transistor due to voltage dividing effect across the access transistor and drive transistor. Moreover, the voltage transfer gain of the cell inverter is lowered due to the parallel connection of the access transistor and the load transistor. This severely deteriorates the cell immunity to the noise. If the (a)

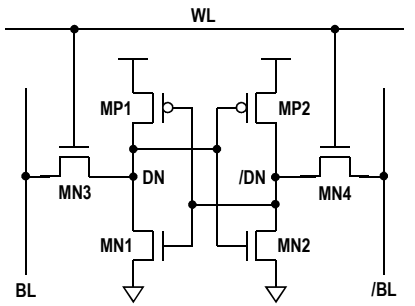

(b)

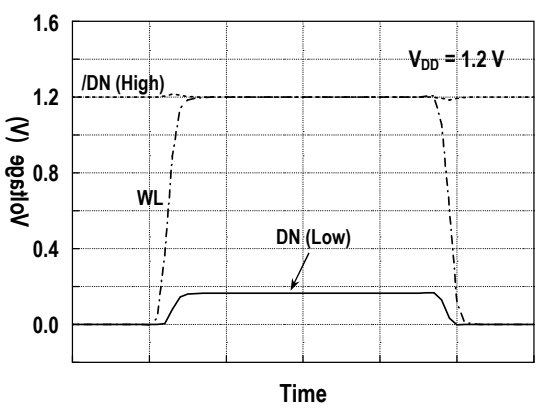

Fig. 1. Standard 6T SRAM cell: (a) schematic; (b) waveforms showing the internal voltage rising in the read operation. (WL: wordline, BL: bitline, /BL: /bitline, DN: data-node, /DN: /data-node)

'low' node voltage is higher than the logic threshold of the other cell inverter, the cell contents may be flipped, resulting in a read failure. For the read functionality, it is desirable to strengthen the drive transistors and weaken the access transistors. Meanwhile, to achieve a proper write operation, an access transistor with strong driving capability and relatively weak load transistor are desired. A careful transistor sizing to ensure a balanced stability for read and write operation is required with right device size to minimize SRAM cell area.

The proposed 8T SRAM cell is shown in Fig. 2(a). In its structure, two PMOS transistors (MP3, MP4) called conducting transistor are added between the access transistor and the drive transistor. Their gates are controlled by a column-wise write-assist line (WAL). In the standby mode, WAL is kept to the ground such that the conducting transistors are normally in on-state. Two cross-coupled inverters (MP1-MN1, MP2-MN2) in the cell hold their bi-stable data. The read operation shown in Fig. 2(b) is basically same as that of the standard 6T cell. Initially consider that $\mathrm{DN}=0$ and $/ \mathrm{DN}=\mathrm{V}_{\mathrm{DD}}$. 
(a)

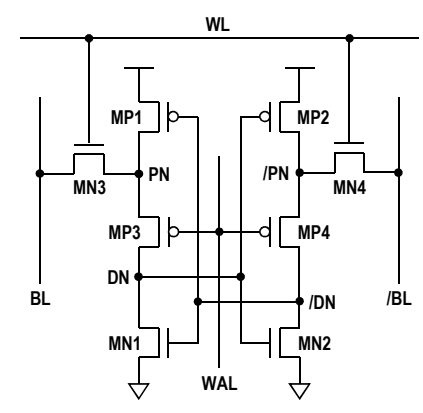

(b)

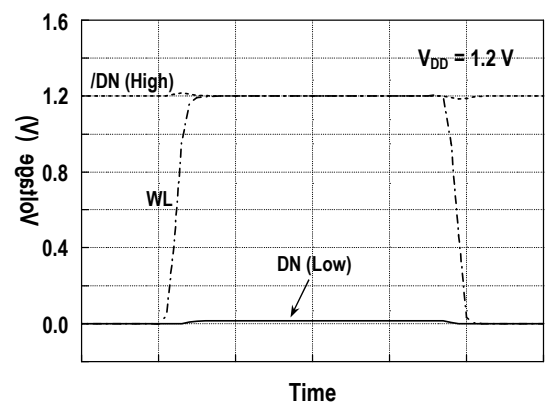

Fig. 2. Proposed 8T SRAM cell: (a) schematic; (b) voltage waveforms for the read operation. (WL: wordline, BL: bitline, /BL: /bitline, WAL: write-assist line, DN: data-node, /DN: /data-node)

At onset of a read access, the bitline pairs are precharged to high, and the write-assist line is tied to the ground. When the wordline is going to high, the voltage dividing in series along access transistor (MN3), conducting transistor (MP3) and drive transistor (MN1) represses voltage rising of internal node DN representing a zero. That leads to increase the read stability drastically. This effect is primarily depending on the threshold voltage of conducting-PMOS transistor. In contrast to the $6 \mathrm{~T}$ cell, the strength of pull-down drive transistor can be reduced in this $8 \mathrm{~T}$ cell. In the write access, the WAL signal is changed to a negative voltage. For a successful write, the bit-cell becomes mono-stable, forcing the internal voltages to the correct values. Initially assume that $\mathrm{DN}=\mathrm{V}_{\mathrm{DD}}$ and $/ \mathrm{DN}=0$. In order to write a zero to the node $\mathrm{DN}, \mathrm{BL}$ is set to ' 0 ' and $/ \mathrm{BL}$ to $\mathrm{V}_{\mathrm{DD}}$. When $\mathrm{WL}$ is going to high, the node $\mathrm{PN}$ makes a transition from $\mathrm{V}_{\mathrm{DD}}$ to a zero. Since the gates of conducting transistors are biased to a negative voltage, the node $\mathrm{DN}$ can be

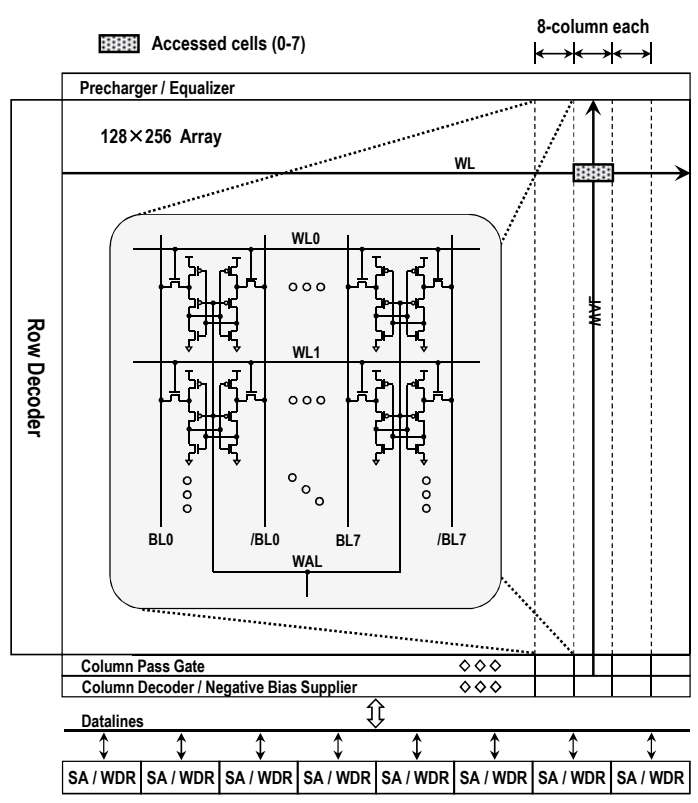

Fig. 3. Configuration of 8T SRAM memory block. (SA: sense amplifier, WDR: write driver)

discharged easily from $\mathrm{V}_{\mathrm{DD}}$ to a zero state. Falling of the node $\mathrm{DN}$ brings the inverter $\mathrm{MP} 2-\mathrm{MN} 2$ to trigger. The positive feedback inside the cell changes the contents of the cell.

Fig. 3 shows an example of $32-\mathrm{kbit}$ SRAM array incorporating the proposed $8 \mathrm{~T}$ cell. The logical organization is basically same as that of the conventional 6T SRAM array. A unique feature is that the negative bias supplier controls the WAL signal running parallel to the bitlines and feeds eight WALs in common. The remaining core circuitry and peripheral logic are essentially same as those for conventional one. For each write cycle, a negative bias supplier decoded by column address activates only eight of interleaved columns. The cells from remaining columns on a selected row will experience a dummy read operation. Moreover, the un-accessed cells on the selected columns will be in the standby mode.

Fig. 4 shows a negative bias supplier and the simulated waveforms associated with a write access using this scheme. The circuit consists of column decoding gates, capacitor driver, boosting capacitor, negative level shifter and pre-bias transistor. A 


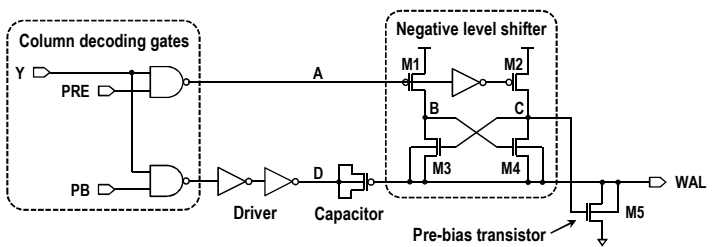

(a)

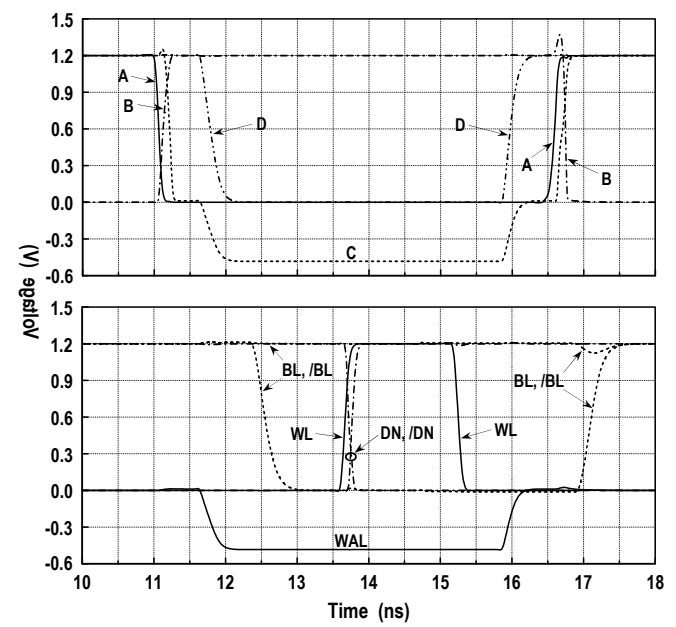

(b)

Fig. 4. Negative bias supplier: (a) circuit configuration; (b) simulated waveforms in the write access at $\mathrm{V}_{\mathrm{DD}}=$ $1.2 \mathrm{~V}$.

triple-well technology is used for the separated-body NMOS transistors (M3, M4, M5). In the initial state, the input node $\mathrm{A}$ of negative level shifter and the node $\mathrm{D}$ of boosting capacitor are both in $\mathrm{V}_{\mathrm{DD}}$ level. Transistors M2 and M3 are on, and M1 and M4 are off, holding the node $\mathrm{C}$ at $\mathrm{V}_{\mathrm{DD}}$. Thus WAL is connected to the ground by the pre-bias transistor M5. At the beginning of the write access, the column pre-decoded signal $Y$ selects a proper local negative bias supplier. Then the preset signal PRE with high transition makes the node A the ground. M1 and M4 are on, and M2, M3 and M5 are off, allowing WAL to float. The succeeding signal $\mathrm{PB}$ changes the node $\mathrm{D}$ to the ground, and thus WAL is boosted to a negative voltage $V_{\text {WAL }}$ by capacitive coupling. Next, the bitlines are driven with the new data. When the wordline is activated later on, the cell data nodes (DN, /DN) flip from one state to another
Table 1. Dimension of SRAM bit-cell transistors.

\begin{tabular}{|c|c|c|c|}
\hline & & $6 \mathrm{~T}$ cell & $8 \mathrm{~T}$ cell \\
\hline \multirow{4}{*}{$\begin{array}{l}\text { Width/Length } \\
\quad(\mathrm{nm} / \mathrm{nm})\end{array}$} & $\begin{array}{l}\text { Load } \\
\text { transistor }\end{array}$ & $150 / 130$ & $150 / 130$ \\
\hline & $\begin{array}{l}\text { Access } \\
\text { transistor }\end{array}$ & $150 / 130$ & $360 / 130$ \\
\hline & $\begin{array}{l}\text { Conducting } \\
\text { transistor }\end{array}$ & - & $150 / 130$ \\
\hline & $\begin{array}{l}\text { Drive } \\
\text { transistor }\end{array}$ & $270 / 130$ & $150 / 130$ \\
\hline \multicolumn{2}{|c|}{ Beta ratio } & 1.8 & 0.42 \\
\hline
\end{tabular}

immediately. After the wordline is switched again to the ground, the signals $\mathrm{PB}$ and PRE return both boosting capacitor and negative level shifter to the initial state. On the other hand, the VWAL level, determined by the ratio of boosting capacitor and parasitic capacitances associated to the WAL node, is $-0.48 \mathrm{~V}$ at $1.2 \mathrm{~V}$ supply, that is, $\mathrm{V}_{\mathrm{WAL}}=-0.4 \mathrm{~V}_{\mathrm{DD}}$ in this example. For the $130 \mathrm{~nm}$ CMOS technology used in this work, the gate oxide thickness is $30 \stackrel{\mathrm{A}}{\text {, }}$ and the breakdown voltages for NMOS and PMOS are $2.5 \mathrm{~V}$ and $-2.5 \mathrm{~V}$ respectively. Since the maximum stress voltage (here $1.68 \mathrm{~V}$ ) on the transistors connected to the WAL node is relatively small, it would not affect the reliability of transistors.

\section{Bit-Cell Performance and Discussions}

\subsection{Bit Area}

For comparisons of stability and leakage properties, the $6 \mathrm{~T}$ and proposed $8 \mathrm{~T}$ cells have been implemented in a $130 \mathrm{~nm}$ generic CMOS technology. The dimensions of each cell transistor are summarized in Table 1 . In the standard $6 \mathrm{~T}$ cell, the width of drive transistor is $270 \mathrm{~nm}$ while those of other transistors are $150 \mathrm{~nm}$. The cell beta ratio, defined as the ratio of $\mathrm{W} / \mathrm{L}$ for driver transistor to $\mathrm{W} / \mathrm{L}$ for access transistor, is equal to 1.8 .

The layouts of the 6T and 8T SRAM cells are shown in Fig. 5. These layouts have been drawn in a pure logic design rule without any cell-based specific rule. In drawing the $8 \mathrm{~T}$ cell, the PMOS and drive transistors layout was first formed with the minimum dimension of the design rules. The access 
(a)

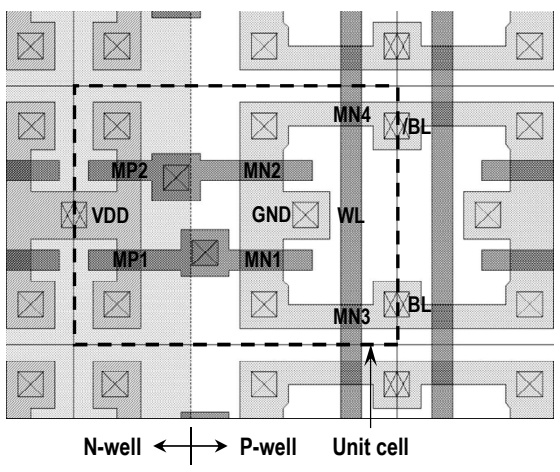

(b)

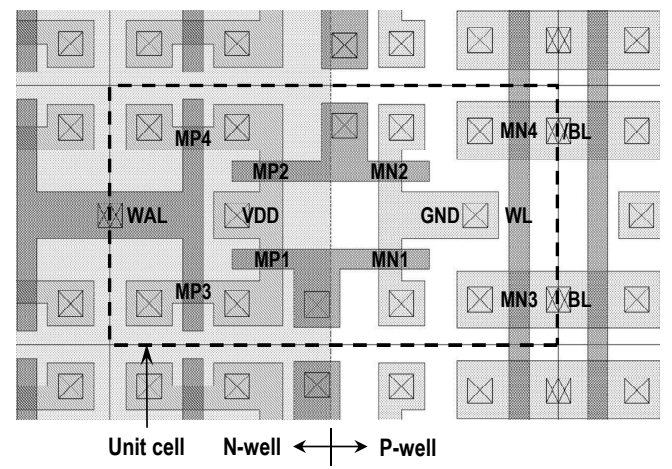

Fig. 5. SRAM cell layouts: (a) standard 6T cell $(1.65 \times 2.06$ $\left.\mu \mathrm{m}^{2}\right)$; (b) proposed $8 \mathrm{~T}$ cell $\left(1.65 \times 2.85 \mu \mathrm{m}^{2}\right)$.

transistor layout was then formed within tolerance of the cell area. The bitline contacts and conducting-PMOS gate contact are shared with neighboring cells. As the result, $\mathrm{W} / \mathrm{L}$ of access transistor is $360 / 130 \mathrm{~nm}$. The other transistors have a minimum device size. The $8 \mathrm{~T}$ bit-cell adds $38 \%$ area overhead relatively to the $6 \mathrm{~T}$ cell. In more scaled CMOS technology such as $65 \mathrm{~nm}, 45 \mathrm{~nm}$ and beyond, the thin cell layout approach [12] might be effective to reduce the lithographic mismatch. But the area overhead may be remained in a same degree since both $6 \mathrm{~T}$ and $8 \mathrm{~T}$ cells will be scaled in asame way.

\subsection{Read Stability}

Fig. 6 shows the simulated butterfly curves for both standard 6T and proposed 8T SRAM cells. The plotted data was obtained using a set of

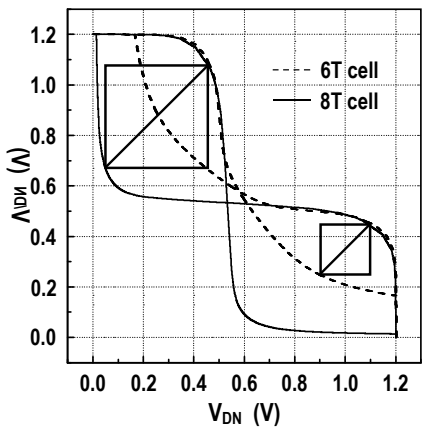

Fig. 6. Simulated butterfly curves at $\mathrm{V}_{\mathrm{DD}}=1.2 \mathrm{~V}$.

(a)

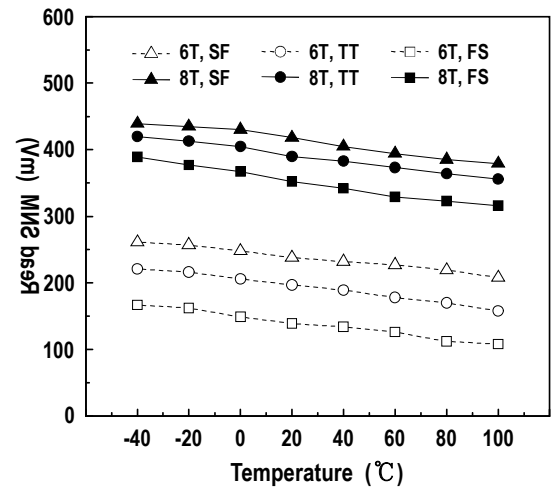

(b)

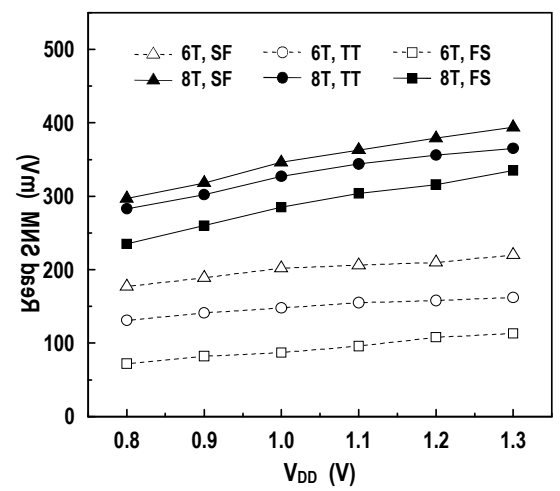

Fig. 7. Read static noise margin: (a) at $\mathrm{V}_{\mathrm{DD}}=1.2 \mathrm{~V}$; (b) at $\mathrm{T}=100{ }^{\circ} \mathrm{C}$.

nominal parameters at $1.2 \mathrm{~V}$ and room temperature. The static noise margin (SNM) is a side of the maximum square in the curve. In spite of a small beta ratio, the SNM of the proposed $8 \mathrm{~T}$ cell is about $100 \%$ larger than that of the standard 6T cell. This is because that the internal node rising 


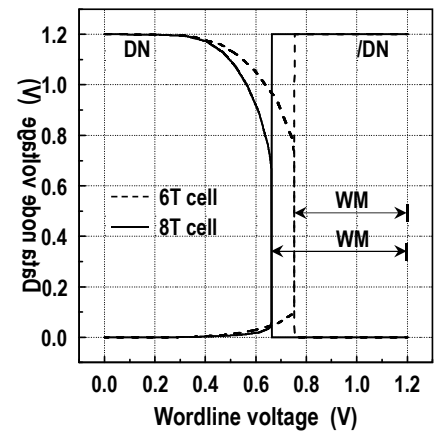

Fig. 8. Simulated write-ability at $V_{D D}=1.2 \mathrm{~V}$ and $V_{\text {WAL }}$ $=-0.48 \mathrm{~V}$.

representing a zero is limited below $\mathrm{V}_{\mathrm{DD}}-\mathrm{V}_{\mathrm{TN}}-$ $\left|V_{T P}\right|$, where $V_{T N}$ and $V_{T P}$ are the threshold voltages of NMOS access transistor and PMOS conducting transistor, respectively. This gives near-ideal butterfly curve essential for robust SRAM bit-cell design.

Fig. 7 shows temperature and $V_{D D}$ dependence on the read stability for different process corners. Here, TT means typical-NMOS and typical-PMOS, FS means fast-NMOS and slow-PMOS, and SF means slow-NMOS and fast-PMOS, etc. In FS (low $\mathrm{V}_{\mathrm{TN}}$ and high $\mathrm{V}_{\mathrm{TP}}$ ) corner, the logic threshold voltage of the cell inverter would be decreased and thus getting the read stability worse. Similarly for $\mathrm{SF}$ (high $\mathrm{V}_{\mathrm{TN}}$ and low $\mathrm{V}_{\mathrm{TP}}$ ) corner, the logic threshold voltage would be increased, getting the read stability better. As the temperature is raised, the read SNM decreases monotonously. At $1.2 \mathrm{~V}$ and $100{ }^{\circ} \mathrm{C}$ worst temperature, the variation in the read SNM between extreme corners (SF, FS) is 63 $\mathrm{mV}$ in the $8 \mathrm{~T}$ cell compared to $100 \mathrm{mV}$ in the $6 \mathrm{~T}$ cell, indicating better process variation tolerance.

\subsection{Write-Ability}

Write-ability of a bit-cell gives an indication of how easy or difficult it is to write to the cell. The write margin (WM) in this work defines the difference between $\mathrm{V}_{\mathrm{DD}}$ and a wordline voltage needed to flip the cell content when the bitline pairs are set with $\mathrm{V}_{\mathrm{DD}}$ and $0 \mathrm{~V}$ [13]. The larger the voltage difference, the easier it is to write to the cell. (a)

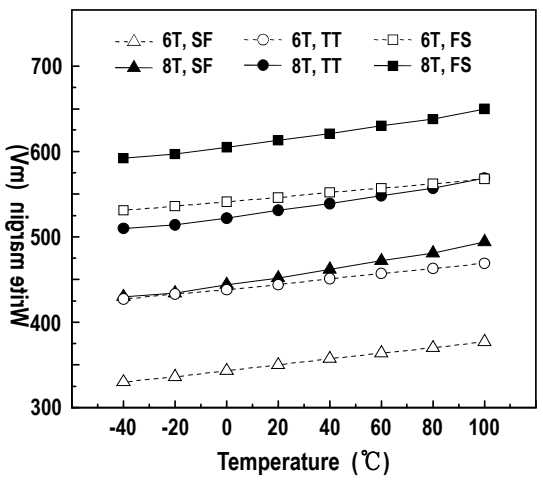

(b)

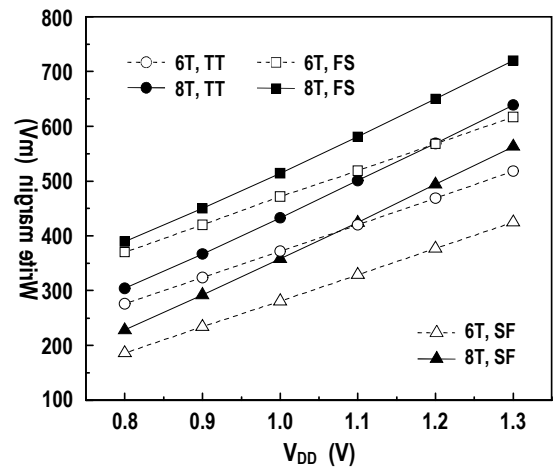

Fig. 9. Write margin: (a) at $\mathrm{V}_{\mathrm{DD}}=1.2 \mathrm{~V}$ and $\mathrm{V}_{\mathrm{WAL}}=$ $-0.48 \mathrm{~V}$; (b) at $\mathrm{T}=100{ }^{\circ} \mathrm{C}$ and $\mathrm{V}_{\mathrm{WAL}}=-0.4 \mathrm{~V}_{\mathrm{DD}}$.

Fig. 8 shows the simulated write-ability at 1.2 $\mathrm{V}$, TT and room temperature. The plot for $8 \mathrm{~T}$ cell was obtained with a WAL bias VWAL of $-0.48 \mathrm{~V}$. The $\mathrm{WM}$ values are $446 \mathrm{mV}$ and $537 \mathrm{mV}$ for $6 \mathrm{~T}$ and proposed $8 \mathrm{~T}$ cells, respectively. Unlike the $6 \mathrm{~T}$ cell, the strength of access transistor can be increased in the $8 \mathrm{~T}$ bit-cell within tolerance of bit area. Owing to the negatively-biased conducting transistor with a strong access transistor, the node storing ' 1 ' in the $8 \mathrm{~T}$ cell is flipped at a much lower WL voltage, giving a higher WM. Compared to the $6 \mathrm{~T}$ cell, the $8 \mathrm{~T}$ cell gives better read stability as well as better write-ability.

Fig. 9 shows the write margin simulation results across temperature and for different supply voltages. The write-ability is getting better at a high temperature because the PMOS load transistor weakens relatively to the NMOS access transistor 
(a)

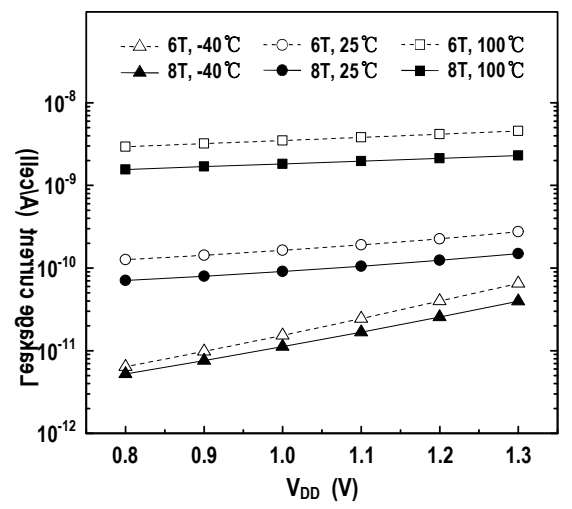

(b)

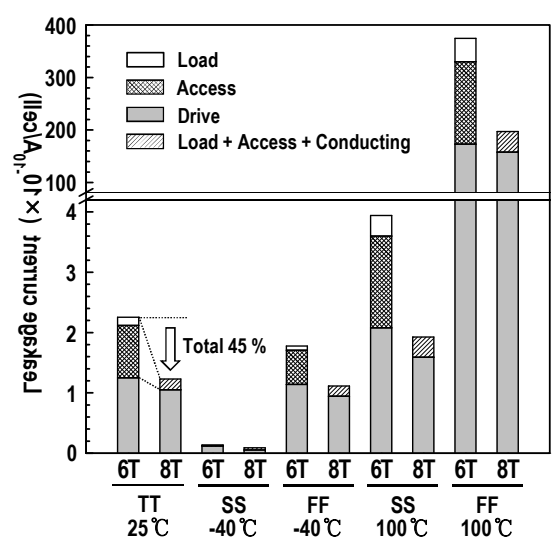

Fig. 10. Standby cell leakage current: (a) at TT process corner; (b) at $\mathrm{V}_{\mathrm{DD}}=1.2 \mathrm{~V}$.

as temperature rises. As $V_{D D}$ increases, the write margin also increases. A negative bias on the conducting transistor during write transition enables the $8 \mathrm{~T}$ cell to achieve better write-ability in low supply voltage. As the result, the WM of $8 \mathrm{~T}$ cell remains higher over the $6 \mathrm{~T}$ cell across all of the voltage ranges. Moreover, the variation in WM across skewed process corners (FS, SF) at $1.2 \mathrm{~V}$ and $-40{ }^{\circ} \mathrm{C}$ is $162 \mathrm{mV}$ in the $8 \mathrm{~T}$ cell compared to $201 \mathrm{mV}$ in the $6 \mathrm{~T}$ cell, showing better process variation

tolerance.

\subsection{Leakage Power Consumption}

The proposed bit-cell also lowers the total leakage power. For both 6T and 8T SRAM cells in the standby mode, the access transistors are cutoff and the bitline pairs are charged to $\mathrm{V}_{\mathrm{DD}}$. The gates
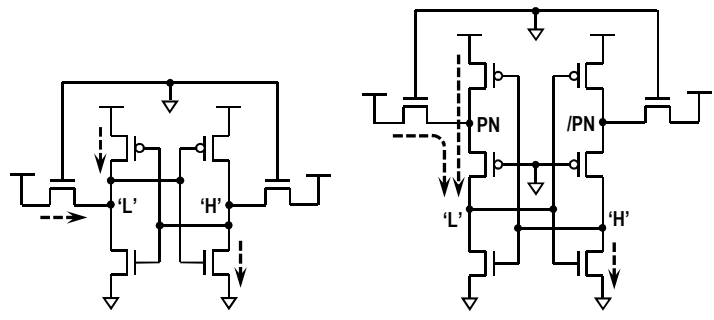

Fig. 11. Subthreshold leakage current path in the $6 \mathrm{~T}$ and 8T SRAM cells.

of conducting transistors in the $8 \mathrm{~T}$ cell are tied to the ground. For each cell, the total leakage current is sum of the subthreshold leakage, the gate induced drain leakage (GIDL), the gate tunneling leakage and the junction leakage of all transistors.

Fig. 10 shows the HSPICE simulated standby leakages of the $6 \mathrm{~T}$ and proposed $8 \mathrm{~T}$ cells, using BSIM4.5 model [14]. As shown in Fig. 10(a), this current increases exponentially as the supply voltage increases. As the temperature is raised, the leakage current also increases exponentially. At $1.2 \mathrm{~V}$ and room temperature, the leakage current consumed by the $8 \mathrm{~T}$ cell is $45 \%$ lower compared to the standard 6T cell. The graph of Fig. 10(b) shows the leakage comparisons consumed at each cell transistor. The small drive transistor contributes to this standby leakage reduction, but main contribution comes from the reduction of the access and load transistor leakage. As illustrated in Fig. 11, the internal node $\mathrm{PN}$ in the $8 \mathrm{~T}$ cell rises to a certain voltage higher than the ground during the standby mode. In this situation, the leakage paths from the access and load transistors to the data 'low' node suffer the stack effect by the conducting-PMOS transistor. Furthermore, the access transistor in the $8 \mathrm{~T}$ cell turns off harder because of the negative $V_{G S}$ and the body effect. The net results reduce the subthreshold component of the leakage drastically. It is more pronounced at high temperature, shown in Fig. 10(a). Likewise the 6T SRAM, this leakage current in the $8 \mathrm{~T}$ cell can be reduced further by introducing the leakage reduction techniques such as the virtual cell ground adjustment [15]. 


\section{Summary}

The stability and leakage power of SRAMs have become an important issue with scaling of CMOS technology. In this paper, a novel 8T SRAM cell realized with the addition of two extra PMOS transistors has been presented. A detailed is $45 \%$ less because of the built-in stacked leakage path.

Using a $130 \mathrm{~nm}$ CMOS technology, the effectiveness of the proposed SRAM cell, which can be applicable to the deep scaled CMOS technology, has been verified under various process, voltage and temperature variations.

Table 2. Comparison of various SRAM bit-cells.

\begin{tabular}{|c|c|c|c|c|c|c|c|c|c|c|}
\hline & Ref. [1] & Ref. [2-4] & Ref. [5] & Ref. [6] & Ref. [7] & Ref. [8] & Ref. [9] & Ref. [10] & Ref. [11] & This work \\
\hline Cell type & $7 \mathrm{~T}$ & $8 \mathrm{~T}$ & $9 \mathrm{~T}$ & $10 \mathrm{~T}$ & $10 \mathrm{~T}$ & $8 \mathrm{~T}$ & $8 \mathrm{~T}$ & $10 \mathrm{~T}$ & $10 \mathrm{~T}$ & $8 \mathrm{~T}$ \\
\hline Reading scheme & $\begin{array}{l}\text { single-- } \\
\text { ended }\end{array}$ & $\begin{array}{l}\text { single-- } \\
\text { ended }\end{array}$ & differential & $\begin{array}{l}\text { single- }^{-} \\
\text {ended }\end{array}$ & $\begin{array}{l}\text { single- }^{-} \\
\text {ended }\end{array}$ & differential & differential & differential & differential & differential \\
\hline Writing scheme & $\begin{array}{l}\text { single- }^{-} \\
\text {ended }\end{array}$ & differential & differential & differential & differential & differential & differential & differential & differential & differential \\
\hline $\begin{array}{l}\text { Efficient } \\
\text { column-interleaving } \\
\text { in write operation }\end{array}$ & possible & impossible & impossible & impossible & impossible & possible & possible & possible & possible & possible \\
\hline $\begin{array}{l}\text { Cell area overhead } \\
\text { over 6T cell }\end{array}$ & $13 \%$ & $33 \%$ & $37.8 \%$ & $66 \%$ & - & $52 \%$ & $36 \%$ & $110 \%$ & $\sim 114 \%$ & $38 \%$ \\
\hline $\begin{array}{l}\text { Read margin } \\
\text { enhancement over } \\
\text { 6T cell } \\
\end{array}$ & increased & $76 \%$ & $100 \%$ & increased & $\begin{array}{l}\sim 100 \% \\
@ 0.8 \mathrm{~V}\end{array}$ & $\begin{array}{l}\sim 60 \% \\
@ 0.6 \mathrm{~V}\end{array}$ & $\sim 100 \%$ & $56 \%$ & $\begin{array}{l}186 \% \\
@ 0.3 \mathrm{~V}\end{array}$ & $100 \%$ \\
\hline \begin{tabular}{|l|} 
Write margin \\
enhancement over \\
6T cell
\end{tabular} & decreased & $0 \%$ & $0 \%$ & $0 \%$ & $\begin{array}{c}0 \% \\
@ 0.27 \mathrm{~V}\end{array}$ & increased & $\begin{array}{l}9.5 \% \\
@ 1.2 \mathrm{~V}\end{array}$ & $\sim 6 \%$ & - & $20 \%$ \\
\hline $\begin{array}{l}\text { Leakage current } \\
\text { over 6T cell }\end{array}$ & - & increased & $\begin{array}{l}22.9 \% \\
\text { reduced }\end{array}$ & $\begin{array}{c}16 \% \\
\text { increased }\end{array}$ & increased & - & - & $\begin{array}{l}18 \% \\
\text { reduced }\end{array}$ & $\begin{array}{c}\sim 10 \% \\
\text { increased }\end{array}$ & $\begin{array}{l}45 \% \\
\text { reduced }\end{array}$ \\
\hline
\end{tabular}

comparison on the results of the cell architecture presented here with the other ones reported in the literature is summarized in Table 2.

The proposed $8 \mathrm{~T}$ cell incorporates a differential read and write path suitable for high-performance mobile applications. In addition, the cell allows an efficient column-interleaving structure for soft-error immunity. It consumes $38 \%$ larger area compared to the standard 6T SRAM cell. In the read operation, the proposed cell represses the data 'low' node voltage rising, thereby enhancing the read static noise margin by $100 \%$ compared to the $6 \mathrm{~T}$ cell. The cell also exhibits $20 \%$ higher write margin with the aid of column-wise negative bias control. The bit-cell itself bears an improved variability tolerance that gives much tight stability distribution across skewed process corners. Furthermore, the leakage power of the idle $8 \mathrm{~T}$ cell

\section{References}

[1] K. Takeda et al., "A read-staticnoise-margin-free SRAM cell for low-VDD and high-speed applications", IEEE Journal of Solid-State Circuits, vol. 41, pp. 113-121, January 2006.

[2] L. Chang et al., "Stable SRAM cell design for the $32 \mathrm{~nm}$ node and beyond", in Symposium on VLSI Technology, Japan, pp. 128-129, 2005.

[3] N. Verma and A. P. Chandrakasan, "A 256 kb 65 nm $8 \mathrm{~T}$ subthreshold SRAM employing sense-amplifier redundancy", IEEE Journal of Solid-State Circuits, vol. 43, pp. 141-149, January 2008.

[4] L. Chang et al., "An 8T-SRAM for variability tolerance and low-voltage operation in high-performance caches", IEEE Journal of Solid-State Circuits, vol. 43, pp. 956-963, April 2008. 
[5] Z. Liu and V. Kursun, "Characterization of a novel nine-transistor SRAM cell", IEEE Transactions on Very Large Scale Integration (VLSI) Systems, vol. 16, pp. 488-492, April 2008.

[6] B. H. Calhoun and A. P. Chandrakasan, "A 256-kb 65-nm sub-threshold SRAM design for ultra-low-voltage operation", IEEE Journal of Solid-State Circuits, vol. 42, pp. 680-688, March 2007.

[7] T. -H. Kim, J. Liu, J. Keane, and C. H. Kim, "A $0.2 \mathrm{~V}, 480 \mathrm{~kb}$ subthreshold SRAM with $1 \mathrm{k}$ cells per bitline for ultra-low-voltage computing", IEEE Journal of Solid-State Circuits, vol. 43, pp. 518-529, February 2008.

[8] M. -F. Chang et al., "A differential data-aware power-supplied $\left(\mathrm{D}^{2} \mathrm{AP}\right)$ 8T SRAM cell with expanded write/read stabilities for lower VDDmin applications", IEEE Journal of Solid-State Circuits, vol. 45, pp. 1234-1245, June 2010.

[9] Y. Chung and D. -Y. Lee, "Differential-read symmetrical 8T SRAM bit-cell with enhanced data stability", Electronics Letters, vol. 46, pp. 1258-1260, September 2010 .

[10] J. P. Kulkarni, K. Kim, and K. Roy, "A 160 mV robust Schmitt trigger based subthreshold SRAM", IEEE Journal of Solid-State Circuits, vol. 42, pp. 2303-2313, October 2007.

[11] I. J. Chang et al., "A $32 \mathrm{~kb} 10 \mathrm{~T}$ sub-threshold SRAM array with bit-interleaving and differential

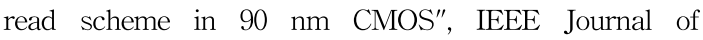
Solid-State Circuits, vol. 44, pp. 650-658, February 2009.

[12] M. Khare et al., "A high performance 90nm SOI technology with $0.992 \mu \mathrm{m}^{2}$ 6T-SRAM cell", in International Electron Devices Meeting, USA, pp. 407-410, 2002.

[13] J. Wang, S. Nalam, and B. H. Calhoun, "Analyzing static and dynamic write margin for nanometer SRAMs", in International Symposium on Low Power Electronics and Design, India, pp. 129-134, 2008.

[14] X. J. Xi et al., "BSIM4.5.0 MOSFET Model User's Manual", Department of Electrical Engineering and Computer Science, University of California, Berkeley, USA, 2005.

[15] Y. Wang et al., "A $1.1 \mathrm{GHz} 12 \mu \mathrm{A} / \mathrm{Mb}$-leakage SRAM design in $65 \mathrm{~nm}$ ultra-low-power CMOS technology with integrated leakage reduction for

mobile applications", IEEE Journal of Solid-State Circuits, vol. 43, pp. 172-179, January 2008.

\section{BIOGRAPHY}

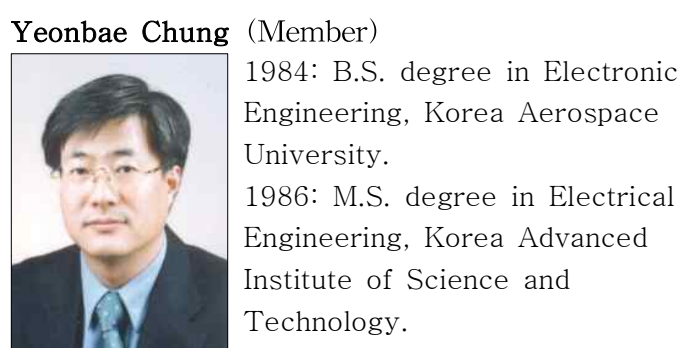

1995: Ph.D. degree in Electrical and Computer Engineering, University of Florida, USA.

1986 1990: Research Engineer at the

Electronics and Telecommunications Research Institute.

1995 2000: Principal Engineer at the Samsung Electronics Corporation.

2000 2002: Project Manager at the Ramtron International Corporation, USA.

2002 Present: Faculty of the Kyungpook

National University.

<Research interest> Embedded memory design, Analog integrated circuits, Low-power VLSI system 